\title{
The Markov Chain of Energy Carryover Reserves in Models of Power Supply Reliability in Remote
}

\author{
Elena Gubiy \\ Melentiev Energy Systems Institute SB RAS, Department of Applied Mathematics, 664033 Irkutsk, Lermontov st., 130, Russia
}

\begin{abstract}
We consider mathematical models for analyzing the energy supply reliability of isolated systems and propose a three-level complex of nested models. The lower level represents the model of functioning of the energy supply system during the period under review. The second level is a model of the energy supply reliability analysis. This analysis is based on multiple simulations of functioning of the energy supply system in randomly formed conditions. The energy sources demand and supply, as well as the amount of carryover reserves of energy in storage, are assumed to be random values. To simulate functioning, the values of energy demand and production are formed using the Monto-Carlo method following their laws of probability. The random value of the carryover reserves is formed using the algorithm that generates the Markov sequence of these reserves. The upper level is represented by the model for selecting the optimal composition of the means ensuring reliability, i.e. energy reserves in the energy production and storage capacity. It was revealed that the algorithm for generating the random value of the energy sources carryover reserves yields the homogenous Markov sequence. Sufficient conditions for uniqueness of the stationary state were determined. Based on the experimental calculations, we estimated the number of iterations required to reach the stationary ergodic state.
\end{abstract}

\section{Introduction}

The issue of energy supply reliability for remote settlements is of particular importance and requires a special study. There are several reasons for that. First, fluctuations in the demand and production of energy resources depend on random factors, which stipulates development of special models adapted for research in random conditions. Second, damages caused by shortages are much bigger than in areas close to transport routes due to the limited conditions for maneuvering, haulage, and, in some cases, the limited timing for energy resources delivery and poorly developed transport infrastructure.

This paper presents the models developed to analyze and optimize the efficiency and reliability of energy supply to remote communities from sources subject to strong influences of random factors. This property is typical for energy supply systems operating on renewable sources (e.g., systems based on the biomass grown on energy plantations, local wind and solar electric power systems).

Significant deviations in the production of energy from renewable energy sources may be caused be fluctuations of natural and meteorological conditions (air temperature, wind force, solar radiation). Significant deviations from the average expected energy demand can also take place. They may be caused by changes in climatic conditions, production needs, etc. Adverse conditions created by increased demand or reduced volume of energy production may result in shortages accompanied by economic damages that happen, for example, due to the need for expensive emergency supplies of additional energy from remote sources or the use of electricity from expensive sources (e.g. diesel power plants).

In addition to these two random exogenous indicators, a third random factor should also be taken into consideration - the amount of carryover reserves of energy. If during one of the periods the energy consumption is lower than the average value and (or) the volume of energy production is higher than the average expected value, then energy production in this period may exceed the demand. Then the difference between the volume of production and consumption goes as a reserve for the next period. For local energy supply systems, electric energy accumulators can be used as such energy storage devices.

Otherwise, when consumption exceeds production, the available reserves are used. If these reserves are exhausted, then the resulting shortage can be covered by more expensive energy resources. For local energy supply systems, diesel power plants can be used as such additional sources. Boiler and furnace fuel supply systems use long-haul fuel.

As a means of ensuring the energy supply reliability, we consider creation of capacity reserves in the production of energy resources from the source under study $\gamma$ and creation of storage tanks with storage 
capacity $z$ for carryover reserves. Values $\gamma$ and $z$ are the optimized indicators. Both of these variables are assumed to be dimensionless, measured in fractions of the mathematical expectation for the energy demand per the unit of time in question.

Let $\bar{Q}$ be the mathematical expectation of the energy demand per unit of time and $\bar{R}$ be the mathematical expectation of the energy production per unit of time. The capacity reserve is measured as a relative excess of the mathematical expectation of production over the mathematical expectation of demand per unit of time:

$$
\gamma=(\bar{R}-\bar{Q}) / \bar{Q}
$$

We consider the algorithm of forming carryover reserves of energy, which can be interpreted as an imitation of a long-term change in reserves as a result of random realizations of production and demand in each period.

\section{Random factors affecting reliability}

Our model takes into account three random variables: average annual demand, average annual production volume, and energy resources carried over from one period to another. The first two random variables are implemented directly by the Monte Carlo method; the third one is produced iteratively during the most repeated simulation of the functioning of the energy supply system.

\subsection{Random energy demand variable}

The demand of settlements for energy, especially in rural areas, is largely associated with domestic heating problems. Therefore, random deviations of energy demand $Q_{v}$ are mainly caused by deviations in the duration of heating periods and average winter temperatures. Using the data on the average daily outdoor temperature [1] accumulated over many decades we can determine the possible deviations of demand for energy resources.

Let $\tau=1, \ldots, T$ be the numbers of the past heating periods with the available data on average daily temperature. Denote by $N_{\tau}$ the duration of the heating period $\tau$ in days. In the calculations presented below, we assume that the heating period starts/ends after the average daily air temperature stayed below/above $8{ }^{\circ} \mathrm{C}$ for five days in a row $[2 ; 3]$.

To describe possible fluctuations in energy consumption, we will use deviation of energy demand for heating during one of the heating periods from the average expected level

$$
b_{\tau}=B_{\tau} / \bar{B}, \tau=1, \ldots, T,
$$

where $B_{\tau}$ is the indicator of the integral temperature difference for the heating period calculated by the formula

$$
B_{\tau}=\sum_{\chi=1}^{N_{\tau}}\left(\hat{t}-t_{\tau \chi}\right), \tau=1, \ldots, T
$$

$\bar{B}$ is the average for all considered heating periods integral difference between the indoor and outdoor temperatures:

$$
\bar{B}=\frac{1}{T} \sum_{\tau=1}^{T} B_{\tau},
$$

Here $\hat{t}$ is the standard indoor temperature; $t_{\tau \chi}$ is the average outdoor temperature on the day $\chi$ during the heating period $\tau$.

In the model of energy supply reliability analysis [4; 5], the random variable generation for the energy resources production is performed using a random number sensor. This sensor selects one of the values from the past periods of observations for the relative deviations of the integral temperature difference $b_{\tau}$, calculated by formula (2). It then calculates a random value of energy demand. Then the random energy demand variable is found as

$$
Q_{v}=b \cdot \bar{Q},
$$

where $b$ is a value of relative deviation of the integral temperature difference randomly selected from the set $b_{\tau}, \tau=1, \ldots, T ; \bar{Q}$ is a mathematical expectation of energy demand.

The boundaries of the interval of possible values $\left[Q^{1}, Q^{2}\right]$ of the random energy demand variable are determined by the rules:

$$
\begin{aligned}
& Q^{1}=\min _{\tau} b_{\tau} \bar{Q} ; \\
& Q^{2}=\max _{\tau} b_{\tau} \bar{Q} .
\end{aligned}
$$

\subsection{Random energy production variable}

Significant deviations in the energy production might occur due to natural and human factors. To estimate possible deviations in energy production and the probability of their realization, we use a truncated normal law with the expertly estimated and varied range of possible values and dispersion

When analyzing the reliability and efficiency of a particular set of backup means, we assume that we are given the mathematical expectation of energy production $\bar{R}$, the standard deviation variable $\sigma$ and the coefficients that determine the interval $\left[R^{1}, R^{2}\right]$ of possible values for a random energy production variable. The boundaries of these intervals are set by the rules

$$
\begin{aligned}
R^{1} & =\delta^{1} \bar{R}, \\
R^{2} & =\delta^{2} \bar{R}
\end{aligned}
$$

The random energy production variable is chosen by the Monte-Carlo method from the interval $\left[R^{1}, R^{2}\right]$ using the probability density function $P(R)$ which stays positive at the specified interval and turns into zero outside of it.

$$
\begin{gathered}
\int_{R=R^{1}}^{R^{2}} P(R) d R=1 ; \\
P(R)>0, R \in\left[R^{1}, R^{2}\right] . \\
P(R)=0, R \notin\left[R^{1}, R^{2}\right] .
\end{gathered}
$$




\subsection{Random variable of the carryover reserves of energy resources energy production variable}

We are not able to estimate realization probabilities for the variable of the energy resources carryover reserves, because they depend not only on the realization probabilities of last year production and demand, but also on the reserves from the year before and, correspondingly, their probabilities. We assume that the probability law for the carryover reserves in the beginning of the specified and the next periods is the same.

\section{The system of nested models for analyzing and optimizing the reliability of energy supply to remote settlements}

To analyze and optimize the means of energy supply reliability for remote settlements, we propose a system of three nested models [4]: simulation model for the power supply system operation during one period; reliability analysis model; model for optimization of energy system reliability.

The mathematical expectation of the annual demand for energy resources $\bar{Q}$ and the specific losses caused by energy shortages $\mu$ are exogenous for calculations.

Simulation model of the energy supply system. Denote by $u_{v}$ the inventory balances of energy resources after the integration $v$. Denote by $v=0, \ldots, V$ the iteration number of the simulation. The value $u_{0} \geq 0$ is given. The amount of reserves carried over to the next period is determined on the basis of the known reserves from the previous period:

$$
s_{v}=\min \left\{u_{v-1} ; z\right\}, v=0, \ldots, V .
$$

This expression takes into account the energy storage capacity $z$.

The available energy is defined as the sum of the produced energy and reserves from the previous period:

$$
R R_{v}=R_{v}+s_{v} .
$$

Shortage at the iteration $v$ is defined by the formula

$$
D_{v}=\left(Q_{v}-R R_{v}\right)_{+} \text {. }
$$

According to (15), the shortage occurs when the demand exceeds the available resources, otherwise the shortage is equal to zero. Here, the function $(x)_{+}$with respect to the real $x$ is a non-negative cut-off function: $(x)_{+}=\max \{0, x\}$.

The balance of energy forms when the energy demand is less than the available energy:

$$
u_{v}=\left(R R_{v}-Q_{v}\right)_{+} \text {. }
$$

The model for analyzing energy supply reliability is based on the repeated imitation of the energy system functioning during a single time period

The simulation model calculates indicators characterizing the functioning of the energy system at each iteration. Upon passing through all iterations, generalized indicators of reliability and average energy supply costs are calculated.

1. The mathematical expectation of shortage

$$
M D=\frac{1}{V} \sum_{v=1}^{V} D_{v}
$$

2. The shortage probability

$$
P D=\frac{T D}{V},
$$

where $T D$ is the number of samplings when shortage occurs

$$
T D=\sum_{v=1}^{V} \operatorname{sgn}\left(D_{v}\right)
$$

Here

$$
\operatorname{sgn}\left(D_{v}\right)=\left\{\begin{array}{l}
1, \text { если } D_{v}>0 \\
0, \text { если } D_{v} \leq 0
\end{array} .\right.
$$

3. The mathematical expectation of the cost for ensuring the reliability and repair of damage caused by shortages

$$
F(\gamma, z)=\mu M D+\frac{1}{V} \sum_{v=1}^{V} \Delta C_{v}\left(\gamma, z, R_{v}, s_{v}\right),
$$

where $\mu$ are damages per unit (include the cost of stock energy resources).

The upper level of the nested models system is a model for optimizing the means for ensuring the energy supply reliability. In the reliability optimization model, we study and solve a two-parameter optimization problem

$$
\begin{gathered}
F(\gamma, z) \rightarrow \min , \\
\gamma \geq 0, \\
z \geq 0 .
\end{gathered}
$$

The optimization is carried out by comparing values of the function $F(\gamma, z)$ when successively varying the value of each of the arguments (for example, using the coordinate descent method). To solve the problem of one-dimensional optimization, the golden section method can be applied.

\section{Algorithm for changing the random variable of carryover energy reserves}

Calculation of the amount of the carryover energy reserves at the beginning of the next iteration can be represented as the following rule:

$$
s_{v+1}=\min \left\{\left(L_{v}+s_{v}\right)_{+} ; z\right\} \text {. }
$$

Here $L_{v}$ is the difference between the random variables of energy production and consumption

$$
L_{v}=R_{v}-Q_{v} .
$$

This variable $L=R-Q$ is a difference between a variable $R$ with a given continuous probability density (10) and a discrete random variable $Q$. Let $Q_{i}, i=1, \ldots, T$ be the ordered ascending set of values $b_{\tau} \bar{Q}$.

$$
\begin{gathered}
Q_{1}=Q^{1}, Q_{T}=Q^{2} ; \\
Q_{i} \leq Q_{i+1}, i=1, \ldots, T-1 .
\end{gathered}
$$

Since we assume that probabilities of meeting the demands $Q_{i}$ are equal, the probability density $L$ is found based on the probability density $R$ by the formula

$$
\widetilde{P}(L)=\frac{1}{T} \sum_{i=1}^{T} P\left(L+Q_{i}\right) .
$$


The random variable $L$ may have a positive probability density only on the interval $\left[L^{1}, L^{2}\right]$, where

$$
\begin{aligned}
& L^{1}=R^{1}-Q^{2} ; \\
& L^{2}=R^{2}-Q^{1} .
\end{aligned}
$$

Further, we suppose that

$$
R^{2}-R^{1} \geq \max \left(Q_{i+1}-Q_{i}\right), i=1, \ldots, T-1 .
$$

In other words, the variables $Q_{i}$ are located densely enough. Then, according to (10), (27)-(29), the random variable $L$ has a positive probability density on the whole interval $\left[L^{1}, L^{2}\right]$ :

$$
\begin{gathered}
\int_{L=L^{1}}^{L^{2}} \widetilde{P}(L) d L=1 ; \\
\widetilde{P}(L)>0, L \in\left[L^{1}, L^{2}\right] ; \\
\widetilde{P}(L)=0, L \notin\left[L^{1}, L^{2}\right] .
\end{gathered}
$$

On the basis of (33), introduce a probability density function for all theoretically possible changes of reserves in the range from $s_{v} \in[0, z]$ to $s_{v+1} \in\left[s_{v}+L^{1}, s_{v}+L^{2}\right]$

$$
\widetilde{\varphi}\left(s_{v+1}, s_{v}\right)=\widetilde{P}\left(s_{v+1}-s_{v}\right) \text {. }
$$

Here we say "theoretically possible", because in the specified interval $s_{v+1}$ might be negative, if $s_{v}+L^{1}<0$, and might exceed the energy storage capacity, if $s_{v}+L^{2}>z$. The function $\tilde{\varphi}$ is an auxiliary tool for defining the probability density function of transition from the reserves $s_{v}$ to the reserves $s_{v+1}$ on the segment $[0, z]$. At the ends of the segment this function takes the probability value.

Using (25) and (36), the probability function of the transition from $s_{v} \in[0, z]$ to $s_{v+1} \in[0, z]$ is determined by the following conditions:

$$
\begin{aligned}
& \varphi\left(s_{v+1}, s_{v}\right)=\widetilde{\varphi}\left(s_{v+1}, s_{v}\right), \text { если } 0<s_{v}<z ; \\
& \varphi\left(0, s_{v}\right)=\int_{L_{1}+s_{v}}^{0} \varphi\left(x, s_{v}\right) d x, \text { если } L^{1}+s_{v} \leq 0 ; \\
& \varphi\left(z, s_{v}\right)=\int_{z}^{L_{2}+s_{v}} \widetilde{\varphi}\left(x, s_{v}\right) d x, \text { если } L^{2}+s_{v} \geq z .
\end{aligned}
$$

Note that in case of (37) the value of function $\varphi$ is a probability density, whereas if (38) and (39) are realized, then the value of $\varphi$ is probability. The exceptions are theoretically possible situations $L^{1}+S_{v}=0$ and $L^{2}+s_{v}=z$, when the values $\varphi\left(0, s_{v}\right)$ and $\varphi\left(z, s_{v}\right)$ are probability densities.

For a given $s_{v}$ from $[0, z]$, the probability density (the probability at the ends of the segment) of $s_{v+1}$ from $[0, z]$ is defined only by the function $\varphi$. Therefore, for any positive $s_{1}$ from $[0, z]$, generated by the rule (25), the stock sequence $s_{v}, v=1,2 \ldots$ will be the Markov chain. Since $\varphi$ does not change at each iteration, this is the homogenous Markov chain.

Further, we denote by $B(s)$ one of the probability density functions of the variable $s \in[0, z]$ with the properties:

$$
\int_{0}^{z} B(s) d s=1, B(s) \geq 0, s \in[0, z], B(s)=0, s \notin[0, z] .
$$

The specific functions $B$ from this class will be denoted using indices and other symbols.

Let $B_{v}$ - be some probability density function of reserves at the iteration $v$. After the iteration $v$, the probability density function $B_{v+1}$ will take the value

$$
B_{v+1}\left(s_{v}+1\right)=\int_{0}^{z} \varphi\left(s_{v+1}, s_{v}\right) B\left(s_{v}\right) d s_{v}, s_{v+1} \in[0, z] .
$$

We represent this transition from $B_{v}$ to $B_{v+1}$ as a mapping $\psi$ :

$$
B_{v+1}=\psi(B v) .
$$

It is known that a homogenous Markov chain converges to stationary (or finite) states $[6,7]$ for any initial distribution of a random variable.

\subsection{Stationary state}

In our case, the existence of stationary state of the Markov sequence means that there exists the function $\bar{B}$ from class (40), such that

$$
\bar{B}=\psi(\bar{B}) .
$$

Herein, it is important to make sure that the stationary state is unique. The homogenous Markov sequence may have several stationary states. Depending on which of these possible stationary states is used to create reserves, statistical tests might yield considerably different calculation results for the model of analysis of energy supply reliability [8].

\subsection{The unique existence condition for stationary state}

Prove that if condition (32) is fulfilled, the stationary state of the random variable of reserves is unique.

Since $R^{1}<R^{2}, Q^{1}<Q^{2}$, then, according to (30), (31) $L^{1}<L^{2}$. Three cases are possible.

Case 1.

$$
L^{1}<L^{2} \leq 0
$$

If this inequality is satisfied, then, according to (8)-(10), the reserves will not be growing at any iteration. They will only decrease and in the stationary state will be at the same zero level.

\section{Case 2. If}

$$
0 \leq L^{1}<L^{2},
$$

then, according to (8), (9), (11), the reserves will not decrease at any iteration, but will only grow. In the stationary state the level of reserves will take the value equal to $z$.

Case 3, The most typical one during the model implementation

$$
L^{1}<0<L^{2} .
$$

In this case the stock level $s_{v} \in[0, z]$ can decrease with a positive probability (if $s_{v}>0$ ) and increase (if $s_{v}<z$ ). The probability density remains positive at any point of the interval of possible decrease $\left[\max \left\{0, L^{1}+s_{v}\right\}, s_{v}\right]$ and at any point of the interval of possible increase 
$\left[s_{v}, \max \left\{z, L^{2}+s_{v}\right\}\right]$. This fact follows from (32)-(39). Therefore, in a finite number of iterations we can get from any point $s_{v} \in[0, z]$ to any point from the interval $[0, z]$ with a positive probability density. This is sufficient for the homogenous Markov chain under study to have a unique stationary state [9]. Additionally, for this stationary state, the probability density of realization of the random variable of reserves $s$ from any point of $[0, z]$ is positive:

$$
\bar{B}(s)>0, s \in[0, z] .
$$

\subsection{Experimental estimate of the required number of iterations}

In order to claim that the estimates obtained do not depend on the choice of the initial reserves, it is necessary to use a number of iterations that would derive a probability density close to a finite one.

The calculations results presented here and below were carried out using a model for analyzing the fuel supply reliability of energy plantations in the remote settlements located in the coastal areas of Lake Baikal $[10]$. We considered a settlement with an average annual fuel demand of 8000 tons of coal equivalent (tce) of standard fuel, which corresponds to an approximate population of 6000 people. We used the meteorological observations data for 100 winters from 1910 to 2010 .

Fig. 1 and 2 present two indicators of the homogeneous Markov sequence calculated on the model for analyzing the fuel supply reliability of energy plantations $[4 ; 10]$ : the arithmetic mean value of the stock level and the standard deviation from the arithmetic mean value. Here we give three different random implementations of the process using different initial stock values. According to Fig. 1, after 6000 iterations, the arithmetic mean values of the reserves diverge by no more than $2 \%$. Standard deviations diverge by less than $2 \%$ after 4000 iterations. Based on these calculations, we can assume that 6 thousand iterations are enough for the iterative process of varying reserves to arrive to a state close to the stationary one for any initial level of reserves. The results of experimental calculations show that 65 thousand iterations are sufficient to obtain stable clear estimates of reliability indicators, the mathematical expectation of operational costs of the fuel supply system, and the mathematical expectation of damages caused by the shortage.

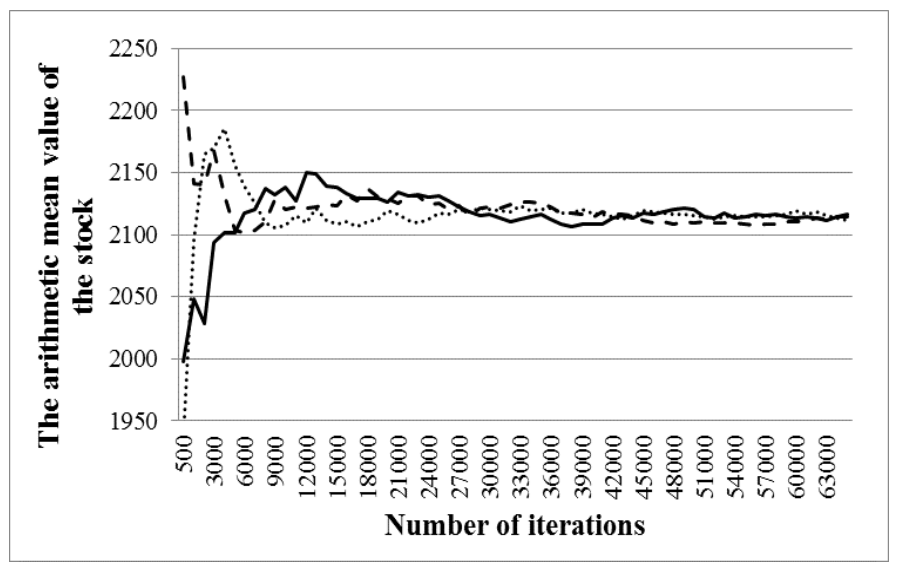

Fig. 1. Dynamics of the arithmetic mean value for the three implementations of the randomly changing carryover reserves

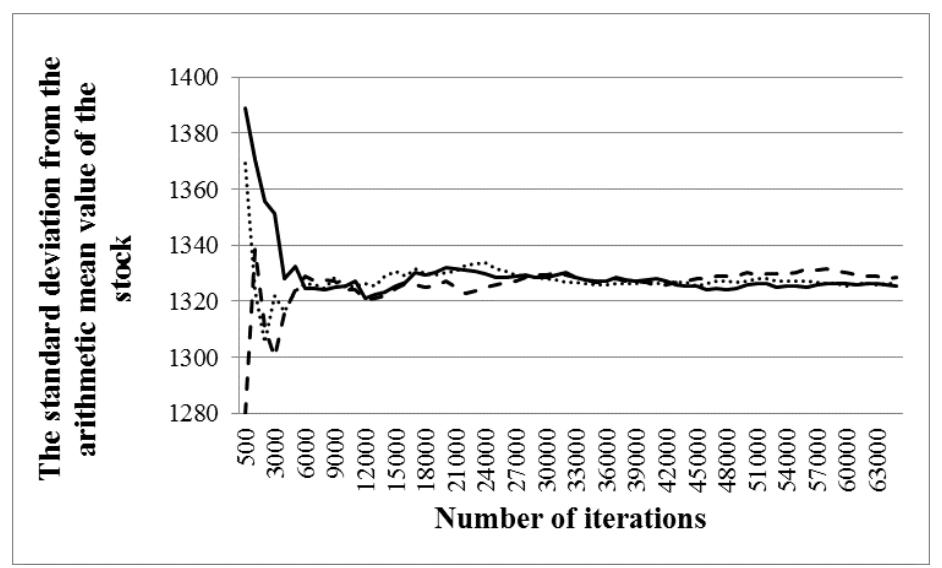

Fig 2. Dynamics of standard deviation from the mean arithmetic value for the three implementations of the randomly changing carryover reserves 
In the example under study, the biggest deviation $Q_{i+1}-Q_{i}$ was 1032 tce. The difference between the biggest and the smallest fuel production output $R^{2}-R^{1}$ was 7986 tce. Therefore, condition (32) is satisfied

\section{Probability density function of reserves for stationary state}

Fig. 3 shows a histogram of the fuel long-term reserves distribution for one of the variants of calculations on the model of fuel supply reliability analysis. The maximum fuel stock capacity is equal to the storage capacity and is 3811 tce. The entire reserves distribution interval is divided into 10 equal subintervals. The values on the abscissa axis correspond to the right boundaries of the subintervals. We can see from Fig. 3 that the increased probabilities of reserves are observed in the first and the last subintervals. The increased probability of reserves in the first subinterval is explained by the increased probability of zero reserves according to (38). The increased probability of reserves in the last subinterval is explained by the increased reserve probability in the storage capacity $z$ (according to (39)). The central part of the histogram is fairly uniform and unimodal.

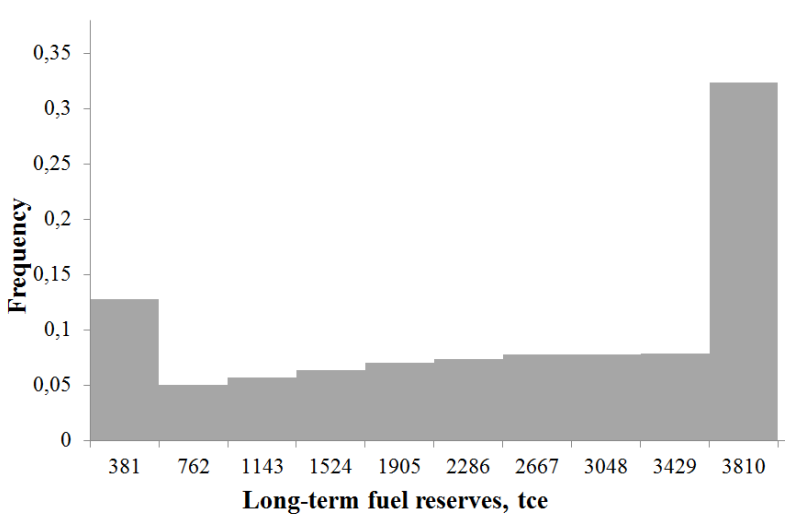

Fig. 3. Histogram of distribution of long-term fuel reserves

Calculations on the model show that with the same probabilities of energy demand and production, the probability distribution of different levels of long-term energy reserves may vary depending on the energy reserve and storage capacity.

\section{Conclusions}

We have developed models for analyzing and optimizing energy supply reliability for remote locations: a simulation model for the functioning of energy supply system; a model for analyzing the energy supply reliability for a given set of stock facilities based on multiple use of the functioning simulation model and the model for optimizing the energy supply reliability based on the variation of stock facilities.

While analyzing and optimizing the energy supply reliability, we account for random factors in the production, consumption, and carryover reserves of energy resources. Realizations of random production and consumption variables in simulation of the functioning of the system are determined by the Monte Carlo method.

It has been shown that the generated sequence of the carryover reserves of energy is a homogenous Markov chain. We have revealed the sufficient condition (32) for the unique stationarity of the random sequence of carryover reserves of energy. This condition can be verified using the input data of the model. Based on multiple experimental calculations, the number of iterations sufficient to obtain clear-cut results has been estimated.

This work was performed within the frame of the Research Project III.17.4.4 of the Fundamental Research Program funded by Siberian Branch of Russian Academy of Sciences No. AAAA-A17-1170303104367 and was financially supported by the Russian Foundation for Basic Research and Government of the Irkutsk region (Grant No. 17-410-380003).

\section{References}

1. O. Bulygina, V. Razuvaev, T. Aleksandrova. Description of the data array on the daily air temperature and precipitation at meteorological stations in Russia and the former USSR (TTTR). URL: http://meteo.ru/it/178aisori.

2. M. Velikanov, A. Nekrasov, A. Reznikovskij, Proceedings of Academy of Sciences. Power Engeneering and Transport, 2, 151-157 (1988).

3. V. Zorkaltcev. Long-term temperature fluctuations and problems of reliability of fuel supply (ESI SB RAS, 2015).

4. E. Gubiy, V. Zorkaltcev. Energy Plantation Efficiency (Nauka, Vovosibirsk, 2018).

5. E. Gubiy, V. Zorkaltcev, Large-Scale Systems Control, 78, 221-234 (2019).

6. D. Bendat, A. Pirsol. Random Data: Analysis and Measurement Procedures (Mir, Moskow, 1971).

7. I. Gihman, A. Skorohod. Theory of random processes (Mir, Moskow, 1971).

8. V. Feller. Introduction to probability theory and its applications (Mir, Moskow, 1984).

9. J. R. Norris. Markov Chains (Cambridge Series in Statistical and Probabilistic Mathematics) (Cambridge University Press, 1998).

10. E. Gubiy, V. Zorkaltcev, Scientific Bulletin of NSTU, 74 (1), 181-196 (2019). 